Classification

Physics Abstracts

$07.80-82.80-61.70 \mathrm{~N}-61.40 \mathrm{~K}$

\title{
Recent Studies of Near-Edge Structure
}

\author{
Lawrence Michael Brown, Caroline A. Walsh, Ann Dray* and Andrew L. Bleloch
}

Cavendish Laboratory, Madingley Rd., Cambridge CB3 OHE, UK

(Received July, 27; accepted December 22, 1994)

\begin{abstract}
The extent to which near-edge structure depends upon long-range order is explored using polymeric systems, where one can vary the disposition of side-groups and the degree of crystallinity and compare the resulting spectra. Carbon K-edges for polystyrene in atactic and isotactic forms are compared; the former is amorphous and the latter crystalline. Differences between the spectra are very slight but observable, and are attributed to bond orientation effects and to interaction between closely-spaced hydrogens bonded to neighbouring benzene rings and to the backbone. The quality of the spectra collected at doses of less than $300 \mathrm{e}^{-} \mathrm{nm}^{-2}$ from a few square microns compares favourably with previously published EXAFS data. Another example of near-edge structure is provided by segregated species. The case of iron segregated to boundaries in $\mathrm{Zr}$ - $\mathrm{Nb}$ alloys is of intense interest to the nuclear industry. An attempt has been made to interpret observed fine structure in terms of structure from reference intermetallic compounds. It seems that the fine structure displayed by the segregated iron is different from that displayed by pure metallic iron or by known compounds with $\mathrm{Zr}$, but more akin to $\mathrm{ZrNbFe}$.
\end{abstract}

\section{Experiments on Isomers of Polystyrene}

A series of experiments was carried out on three different forms of polystyrene in order to investigate the possibility of the effect of long-range order on the electronic structure of polymeric materials. The repeat unit of polystyrene is shown in Figure 1. A benzene ring is attached to every other carbon atom of the carbon backbone. Each carbon atom has two different bonds to which the benzene ring can be attached. In isotactic polystyrene the rings are all attached to the same side of the carbon backbone. This isomer can be produced in both amorphous and crystalline forms. In atactic polystyrene the benzene rings are distributed at random on both sides of the carbon chain, which precludes the possibility of crystallization. Polystyrene was chosen because, by comparison to many polymers, it is less sensitive to irradiation damage by an electron beam. Spectra were acquired from amorphous atactic polystyrene, amorphous isotactic polystyrene and crystalline isotactic polystyrene. The amorphous films were produced by dissolving the polystyrene in toluene and drawing out a film on a glass slide. The crystals were produced as described by Tanzawa [1] and deposited from solution directly onto commercially produced SiO films on $\mathrm{Cu}$

* Deceased 9 October 1994. 
microscope grids; they were characterised by electron microscopy and diffraction. The crystals from which spectra were collected were predominantly oriented with the c-axis approximately parallel to the incident electron beam.

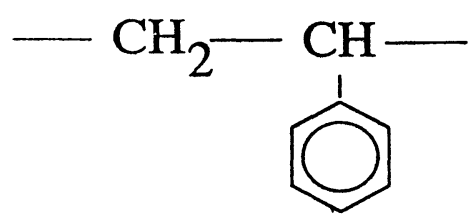

Fig. 1. - The repeat unit in polystyrene.

The spectra were acquired using a parallel EELS spectrometer [2] on a VG HB501 microscope. As it was important to minimise the electron dose given to the specimen, so that the spectra acquired were as close as possible to those from an undamaged specimen, a $25 \mu \mathrm{m}$ objective aperture and a $500 \mu \mathrm{m}$ collector aperture were used. This resulted in a convergence angle of the incident beam smaller than the collection angle, resulting in increased collection efficiency of the scattered electrons, at the expense of some degredation of spectrometer resolution. The spectra were collected whilst scanning an area of $200 \mathrm{~nm} \times 160 \mathrm{~nm}$ for short time intervals, corresponding to an electron dose of $230-330 \mathrm{e}^{-} \mathrm{nm}^{-2}$. After each acquisition the beam was moved to a new area of the sample. The spectra shown in Figure 2 are the result of the addition of many hundred spectra collected as described above and aligned before addition, to compensate for drift in energy, using the sharp $\pi^{*}$ peak (peak A). The resulting spectra were then normalised using the intensity around peak B. It can thus be said that each spectrum comes from a well-defined area of the specimen, a few square microns in size. The dose required to produce the spectrum is about a third of that required to degrade the electron diffraction pattern, as estimated from an independent experiment. The full width at half maximum of peak $\mathrm{A}$ is about $1 \mathrm{eV}$, about twice the spectrometer resolution as estimated from the zero loss peak.

The spectra are presented with the peak $\mathrm{A}$ at $285.5 \mathrm{~V}$ taken as zero. They agree reasonably well with those acquired using X-ray absorption spectroscopy [3]. Most of the peaks can be assigned to empty orbitals in benzene, following Horsley et al. [4]. Thus the spectrum from polystyrene is dominated by its benzene rings. The peak $A$ itself varies considerably in intensity from one sample to the next, but it is always higher in the crystalline case. This effect is largely attributable to the range of orientations of the benzene rings, which is greater in the amorphous material, an effect which has been modelled in some detail [5]. Peak B seems systematically sharper in the crystalline isotactic case than it is in either of the amorphous cases. According to Horsley et al. [4], the oscillator strength for peak B contains three $\sigma^{*}$ states, observed at $8.3 \mathrm{eV}$ in both gaseous and solid benzene; here in polystyrene it appears at almost exactly the same energy. Such states are quite extended, and the width of the band in the solid will be more dependent on long range order than more localised states, and narrower in the crystal than in the amorphous materials, so this may provide a natural explanation for the observations. The closest distance of approach between hydrogens on neighbouring benzene rings in the crystal is in fact less than the spacing between those on the same ring, so this interaction is likely to dominate the overlap integrals. If a comparison is drawn between the amorphous atactic case and the amorphous isotactic case, again the peak B is sharper in the more ordered molecule, as one might expect from this line of argument. 

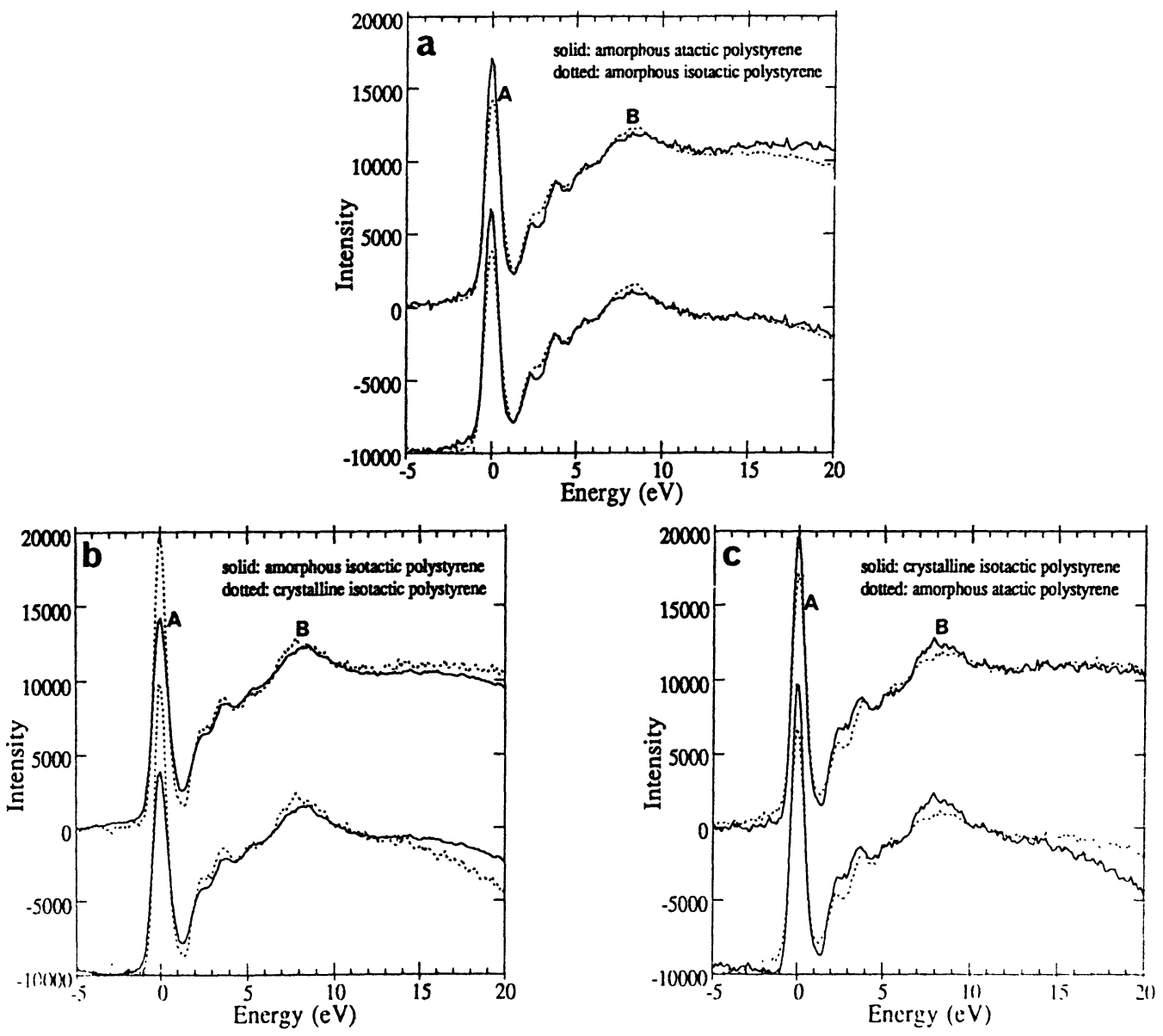

Fig. 2. - Electron Energy Loss spectra from atactic, amorphous isotactic and crystalline isotactic polystyrene., compared in Figures a) to c). The energy zero is taken to be the $\pi^{*}$ peak which is used as a reference in assembling the spectra. The two upper curves in each figure show the raw data. The two lower curves show the data after deconvolution with the valence loss spectra, and have been displaced in intensity. The electron doses and effective collection angles were 250,330 , and $230 \mathrm{e}^{-} \mathrm{nm}^{-2}$ and 4.4, 6.9 and $3.8 \mathrm{mrad}$ for the atactic, amorphous isotactic and crystalline isotactic polystyrene respectively.

\section{Experiments on $\mathrm{Fe}$ in Grain Boundaries of $\mathrm{Zr}-2.5(\mathrm{wt}) \% \mathrm{Nb}$ Alloys}

Interest in this problem arises from CANDU nuclear power reactors, where the presence of trace amounts of iron in the $\mathrm{Zr}-\mathrm{Nb}$ pressure tube at reactor temperatures probably accelerates diffusion by many orders of magnitude, because of anomalous binding between (interstitial) iron and vacancies in the matrix [6]. Although the effect is poorly understood, it is clear that iron is highly segregated to grain boundaries and to interphase boundaries [7] both in irradiated and heat-treated alloys. It is therefore worthwhile trying to detect the fine structure of the segregated iron to deduce if possible something about its chemical state, in particular its $3 \mathrm{~d}$ occupancy. The method used is to compare well- characterised intermetallic compounds with spectra from the segregated species, analysing the fine structure according to the principles of Pearson et al. [8] described below. 
The difficulty is to achieve a robust and reliable numerical method, so that confidence can be placed in the results. Several methods of increasing sophistication were tried, giving qualitatively similar answers but showing quantitative differences.
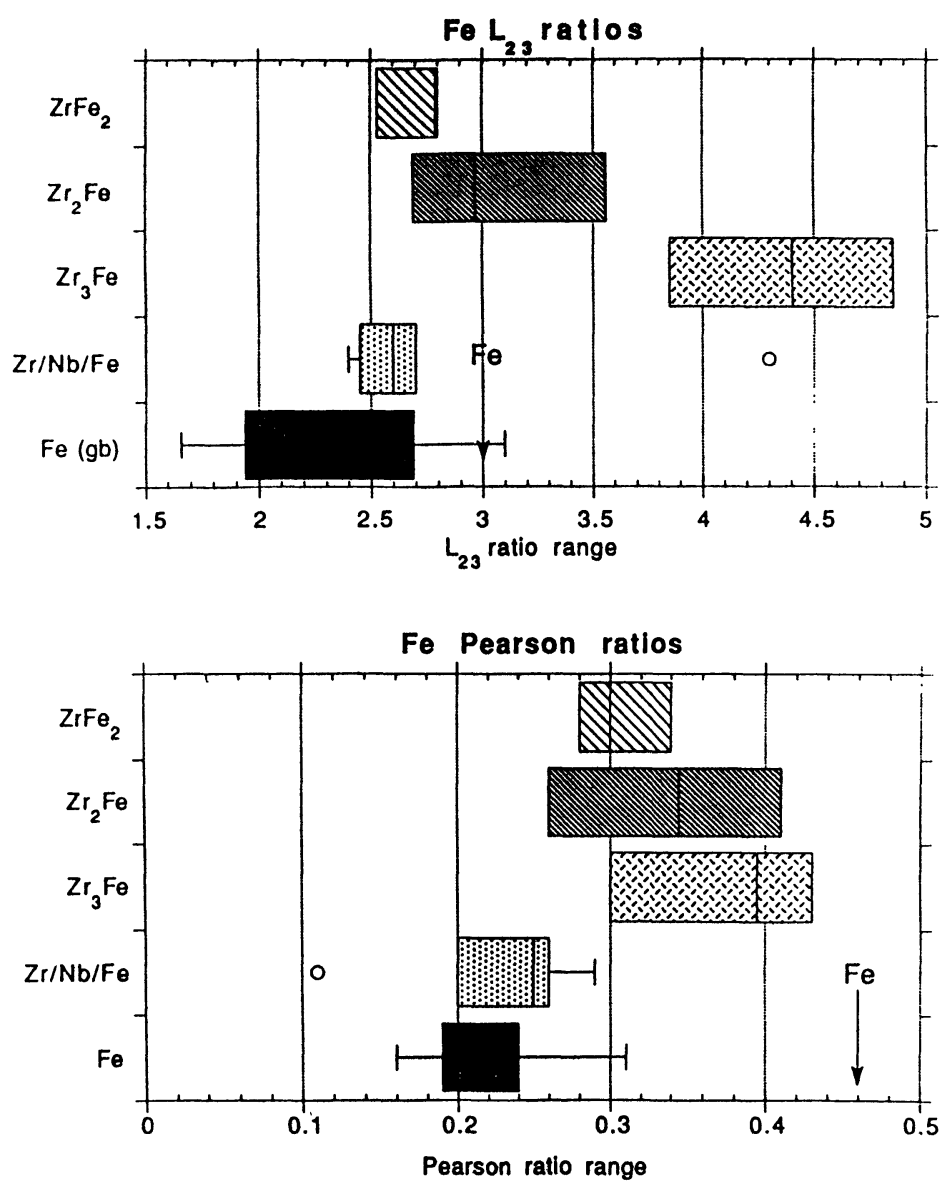

Fig. 3. - Results for segregated iron. The $L_{2}$ to $L_{3}$ intensity ratio (upper diagram) and the ratio of intensity in the white lines to that in the continum ("Pearson ratio") for iron in various intermetallic compounds and (bottom row) for iron segregated to grain boundaries. The vertical line is the mean, and separate rms deviations for readings above and below the mean are shown. The circle is a "rogue" point, not included in the averages. The arrow shows a value for pure metallic iron which agrees well with published data.

A fuller account will be published elsewhere. Briefly, spectra showing the $\mathrm{Fe} L_{23}$ edge at about $710 \mathrm{~V}$ energy loss were acquired at nanometre intervals along a grain boundary in the $\alpha-\mathrm{Zr}$ (a socalled $\alpha-\alpha$ boundary). The alloy contains $1000 \mathrm{ppm}$ Fe overall, an amount too small to analyse except where it is highly concentrated. The alloy had been previously characterised by EDX, and the grain boundaries shown to contain Fe. After dark-current subtraction and flat-field correction, as applied to all spectra acquired using McMullan's CCD system [2], the background was stripped, the white lines were fitted by Gaussians and the continuum by two 'softened' step-functions. The $L_{2} / L_{3}$ ratio and the ratio of the total "white line" intensity to the continuum as sampled by a window $50 \mathrm{~V}$ in width $50 \mathrm{~V}$ above the $L_{3}$ white line onset (the "Pearson ratio") was calculated. Several functions with varying exponents were chosen for the background, and several for the continuum 
rise in the edge. The results felt to be most reliable are shown in Figure 3. The intermetallic compounds were either in the form of homogeneous standards, or well- characterised precipitates. In spite of the scatter and uncertainty concerning precise numerical values, the following statements can be made about the iron segregated to the boundaries:

1) The segregated iron is in a chemical state much more like the ternary intermetallic $\mathrm{ZrNbFe}$ than any other.

2) Because the white-line intensity is lower than that in pure $\mathrm{Fe}$, or in $\mathrm{Fe}$ in any of the binary intermetallics, one can deduce that the segregation is accompanied by electron transfer towards the iron, so that the $d$-bands are fuller than in metallic iron. Consultation with the paper by Pearson et al. [8] suggests that a reduction in the "Pearson ratio" by about 0.2 is caused by the transfer of two electrons to the $3 \mathrm{~d}$ states in the iron, making the segregated state $\mathrm{Fe}^{2-}$. The obvious candidates for transfer are the weakly bound $5 \mathrm{~s}$ electrons of the $4 \mathrm{~d}$ transition elements, of which $\mathrm{Zr}$ has two and $\mathrm{Nb}$ one.

3) The relative filling of the $3 d_{3 / 2}$ and $3 d_{5 / 2}$ states in the iron is unchanged by the charge transfer as indicated by the $L_{2} / L_{3}$ ratio.

\section{Conclusions}

As a result of the greatly improved spectrometers [2], it is now possible to record fine structure from radiation sensitive materials, and from segregated impurities. The combination of electron microscopy and spectrometry would seem to unite the study of crystal defect structure with electronic structure, bringing together chemistry and physics in a satisfying way.

\section{Acknowledgements}

Dr. C.A. Walsh gratefully acknowledges financial support from I.C.I., and helpful suggestions from Drs. R.S. Payne, M. Shannon, R. Crick, and A.M. Donald. Dr. A. Dray thanks Ontario Hydro for financial support, Dr. V. Perovic and Dr. A. Perovic who provided specimens and experience with this problem, and Dr. P. Rez who suggested various numerical procedures. Dr. J. Yuan is thanked for his help, and other members of the Microstructural Physics Group who provide support with the spectrometer.

\section{References}

[1] Tanzawa Y., Polymer 33 (1992) 2659.

[2] McMullan D., Fallon P.J., Ito Y. and McGibbon A.J., in: Proc. 10th European Congr. on Electron Microscopy, Granada, 1992, A. Ríos, J.M. Arias, L. Megías-Megías and A. López-Galindo Eds., Vol. 1, 103.

[3] Outka D.A. and Stöhr J., NEXAFS: A Probe of Local Bonding for Organic Gases, Solids, Absorbates, and Polymers, in: Chemistry and Physics of Solid Surfaces VII, Springer Ser. Surf. Sci. 10, R. Vanselow and R. Howe Eds. (Springer Verlag, 1988).

[4] Horsley J.A., Stohr J., Hitchcock A.P., Newbury D.C., Johnson A.L. and Sette F., J. Chem. Phys. 83 (1985) 6099-6107.

[5] Walsh C.A., "Interpretation of Spectral Features in Electron Energy Loss Spectra of Polymeric Materials", Final Report for SRF Project, available on request from the author (1994).

[6] Hood G.M., Zou H., Schultz R.J, Ray J.A. and Jackman J.A., J. Nucl. Mater. 189 (1992) 226.

[7] Perovic V., Perovic A., Weatherly G.C., Brown L.M., Purdy G.R., Fleck R.G. and Holt R.A., J. Nucl. Mat. (1993) 251.

[8] Pearson D.H., Fultz B. and Ahn C.C., Appl. Phys. Lett. 53 (1988) 1405. See also the review by J.K. Okamoto, D.H. Pearson, C.C. Ahn and B. Fultz, Ch. 8 in Transmission Electron Energy Loss Spectrometry in Materials Science, M.M. Disko, C.C. Ahn and B. Fultz Eds., TMS (Warrendale, Penna, 1992). 